\title{
BMJ Open Implementation of integrated operating rooms: how much time is saved and how do medical staff experience the upgrading? A mixed methods study in Denmark
}

\author{
Kathrine Carstensen, ${ }^{1}$ Emma Kejser Jensen, ${ }^{1}$ Mads Lænsø Madsen, ${ }^{2}$ \\ Anne Marie Ladehoff Thomsen, ${ }^{1}$ Claus Løvschall, ${ }^{1}$ Nasrin Tayyari Dehbarez, ${ }^{1}$ \\ Bettina Wulff Risør (iD ${ }^{1}$
}

To cite: Carstensen K, Jensen EK, Madsen ML, et al. Implementation of integrated operating rooms: how much time is saved and how do medical staff experience the upgrading? A mixed methods study in Denmark. BMJ Open 2020;10:e034459. doi:10.1136/ bmjopen-2019-034459

- Prepublication history and additional material for this paper are available online. To view these files, please visit the journal online (http://dx.doi. org/10.1136/bmjopen-2019034459).

Received 03 October 2019 Revised 28 May 2020 Accepted 15 June 2020
Check for updates

(c) Author(s) (or their employer(s)) 2020. Re-use permitted under CC BY-NC. No commercial re-use. See rights and permissions. Published by BMJ.

${ }^{1}$ DEFACTUM, Central Denmark Region, Aarhus N, Denmark ${ }^{2}$ Department of Procurement \& Clinical Engineering, Central Denmark Region, Aarhus, Denmark

Correspondence to Dr Bettina Wulff Risør; bettriso@rm.dk

\section{ABSTRACT}

Objectives To evaluate staff experiences of the implementation and use of integrated operating rooms (IORs) in comparison to conventional operating rooms (CORs) in Denmark.

Design This study used a mixed methods approach by combining quantitative (registry-based analysis of surgical time) and qualitative (interviews with experienced surgical staff) perspectives.

Setting Hospitals in Denmark.

Methods The quantitative component compared the time consumption of patients between the integrated and CORs in two hospital departments at Aarhus University Hospital. Data were extracted from the administrative system in the hospital. Independent t-tests were used to estimate the statistical differences in the mean time spent on patients between the two operating rooms (ORs), and linear regression was applied to adjust for the potential influence of the surgeon. The explorative qualitative research component involved interviews with 20 informants from 10 hospital departments across seven Danish hospitals, all of whom participated between February and April 2019. Data were analysed using thematic analysis.

Results The quantitative analyses showed that preparation time for lobectomy was significantly lower and completion time for cholecystectomy significantly higher in the integrated compared with CORs. No other statistically significant differences were found. The qualitative analysis showed that some nurses experienced better cooperation with the surgeon and that non-sterile nurses experienced an improved working environment in the integrated compared with CORs. Surgical staff experienced that the IORs led to improved workflow during surgery.

Conclusions This study identified no disadvantages regarding the use of IORs compared with CORs. The quantitative component of the research did not identify convincing statistically significant differences in the time consumption per patient between the ORs and according to the qualitative analyses IORs were not experienced by study participants to lead to major improvements among staff.

\section{Strengths and limitations of this study}

- The mixed method design is a key strength of this study as it provides an in-depth understanding of the consequences of the implementation of integrated operating rooms (IORs) by collecting rich and comprehensive qualitative and quantitative data.

- A strength of the qualitative part of the study was the large and varied sample of interview informants, allowing for exploratory investigation of the different types of use and experienced consequences of the implementation and use of IORs.

- A strength of the quantitative part of the study was the large dataset of time registrations which allowed for statistical analyses of differences in time consumption.

- The study was performed in a Danish hospital setting. By making the organisation and processes within the IORs and conventional operating rooms explicit and transparent, clinicians and researchers in other settings can hopefully assess the relevance and applicability of results to their own context.

- Although not detected in this study, other consequences and value of the IOR may be found over time and in further research studies.

\section{INTRODUCTION}

In 2008, the WHO focused specifically on patient safety in the operating room (OR), launching the campaign 'Safe Surgery Saves Lives' to reduce complications and mortalities arising from the perioperative period. ${ }^{1}$ Unintentional events in the OR are largely related to factors such as surgical competency, team ability to cooperate, problems with surgical equipment or ergonomic deficiencies. ${ }^{2-4}$ In addition, architectural factors in the OR, such as interior design, room size, area exposure, light and acoustic environment, also have an impact on patient safety. ${ }^{5}$ 
The number and complexity of technological systems in the OR have been increasing for many years. ${ }^{6}$ These ongoing technological developments hold new potential for improving safety in the OR. The integrated operating room (IOR) is a comprehensive concept for a group of systems and technologies developed over recent years to support surgical procedures. The IOR is typically used for minimally invasive surgery, that is, endoscopic and laparoscopic procedures, and currently, there are several different systems and suppliers on the market. The IOR brings together the various OR systems and technologies such that the equipment and systems can be operated from a single touch screen that is often placed in the sterile field. The surgeon or surgical nurse can thus access different functions, such as the operating and ceiling lamps, in the OR directly from the touch screen, as well as being able to make continuous and rapid adjustments to the surgical equipment. It is also possible to retrieve information from the electronic patient record, for example, medical record, blood sample responses and imaging material, which can be viewed on the monitors in the sterile field.

There is only limited knowledge available about the consequences of the implementation and use of IORs. Some studies have investigated the importance of overall control from the sterile field and have found that it may result in fewer interruptions during surgery, faster set up of the equipment, better workflow and release of time for the non-sterile nurses. ${ }^{7-10}$ A German study found cost savings using IORs due to reduced time consumption ${ }^{11}$ but these findings were not confirmed in a recent study by Blikkendaal and colleagues, ${ }^{12}$ who, on the contrary, found that the new system created more time-consuming interruptions.

In this study, the time spent on each patient and the staff experiences of implementing and using IORs were evaluated in comparison to conventional operating rooms (CORs) in a Danish hospital setting. We aimed to investigate and describe the differences in procedures between the IOR and COR as well as the consequences of implementing the IOR as experienced by the staff.

\section{MATERIALS AND METHODS}

\section{Technology and the comparator}

The OR, regardless of being an IOR or COR, is typically divided into two to create a sterile and non-sterile field. The sterile field is the work zone for the surgeon and the sterile nurse, while the non-sterile field around this area is the work zone for the anaesthesiologist and the nonsterile nurse.

In this study, an IOR was defined as the functional linkage of a collection of systems and technologies to one unit - a touchpad. The touchpad allows the surgical staff to control all the IOR equipment/functionalities such as the environment (light, temperature and curtains), medical devices and video distribution. The IOR has pre-setting options that can be applied for different types of surgery or the preferences of different surgeons. According to the literature, the touchpad in the IOR is typically placed in the sterile field which allows the sterile surgical staff to interact and control the system. In practice, another touchpad can also be placed in the nonsterile field.

In a COR, the functionalities are often the same as in an IOR but machines and equipment such as lights, curtains, room temperature control, audio/video (A/V) equipment, table, doors and surgical apparatus are controlled by a control panel located in the non-sterile field. This requires the continued presence of a non-sterile nurse to assist the sterile dressed personnel.

\section{Design}

We used a mixed methods approach to obtain an in-depth understanding of the research problem by collecting rich and comprehensive qualitative and quantitative data. ${ }^{13}$ We conducted the study in two phases.

Phase I aimed to identify the activities that varied as a result of differences in the OR set-ups through observational visits in hospital departments that had implemented IOR. Unstructured interviews were performed during the observational visits to ensure that observed activities were properly understood and described. In addition to allowing the identification of procedures that varied between the two OR set-ups, observational visits and unstructured interviews were performed to identify potentially clinically important changes in safety, quality and patient outcomes that might occur as a result of the implementation and use of an IOR.

The results from phase I were used to inform the structure, design and conduct of the quantitative and qualitative studies conducted in phase II. Phase II results were summarised to facilitate an in-depth understanding of the potential consequences and use of IORs. Figure 1 outlines the flow of the study.

\section{Phase I}

Through observational visits and unstructured interviews with the clinical staff in two hospital departments at Aarhus University Hospital (AUH) (see the Setting section), the working procedures in IORs and CORs were carefully described and differences in the existing activities and procedures were identified. The working procedures are illustrated in figures 2 and 3. The identified differences in procedures are written in blue in figure 3 and were used to focus the design of phase II to further investigate these differences quantitatively and qualitatively.

In phase I, the following differences in working procedures between the COR and IOR were identified:

- Differences in the preparation procedures for the OR and surgical equipment.

- Differences in the procedures for the sterile nurses during surgery.

- Differences in the procedures for the non-sterile nurses during surgery. 


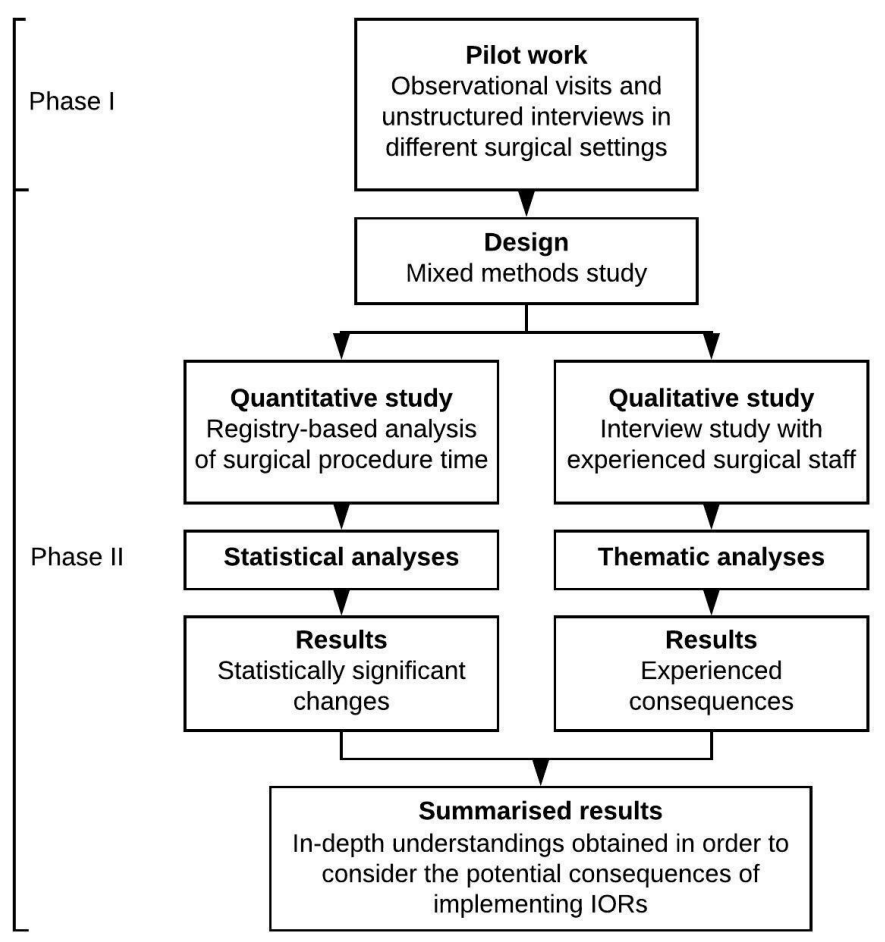

Figure 1 Study flow. IORs, integrated operating rooms.

- Differences in the procedures for cases of acute conversion of the surgery from laparoscopic to open surgery.

Factors such as the pre-setting possibilities for the equipment in the IOR and differences in the procedures for both the sterile and non-sterile nurses resulting in differences in the division of labour during surgery may affect the duration of the surgical procedure. Therefore, time spent on each patient was identified as a quantitative outcome for analysis.

Of the identified differences, acute conversion of the surgery from laparoscopic to open surgery was considered a rare event $(\sim 1$ per month), and thus, consequences related to this procedure were considered of minor importance to the current analysis.

We identified no measurable clinically important changes in the safety, quality or patient outcomes resulting from the implementation and use of the IOR. Therefore, the study did not include such effectiveness outcomes.

\section{Phase II}

Based on the results from phase I, phase II consisted of a quantitative study investigating the time spent on each patient and a qualitative study investigating the experienced consequences of the identified differences in procedures between COR and IOR (see the blue text in figure 3).

\section{Phase II:}

\section{Quantitative study}

Setting

A hospital setting for the quantitative evaluation of IORs was identified based on an assessment of the surgical tasks and availability/implementation of an IOR. Departments were required to have both an IOR and COR and they had to perform comparable surgery at both. The departments were identified within the Central Denmark Region at AUH. Managers of the relevant departments were contacted and accepted to participate in the study. The participating departments were: (1) Department of Heart, Lung and Vascular Surgery and (2) Department of Gastrointestinal Surgery. Surgical staff assisted in identifying the comparable types of surgeries performed in both the IORs and CORs. Table 1 presents the two participating departments along with the identified and included types of surgeries.

Participating departments were recently renewed as part of hospital building and modernisation. Therefore, the CORs (baseline/comparator) were equipped according to the described design guide for standard ORs. ${ }^{5}$ The CORs were, therefore, built ensuring that sufficient space was provided for the equipment to be used and to keep the floor clear of loose cables, and so on. All ORs were built with equipment boom-mounted with the possibility of being positioned ergonomically in relation to the user.

\section{Data collection and analysis}

To measure the surgical procedure time in the IORs and CORs, data were extracted from the clinical booking system at AUH. The following data points were registered by clinicians during every surgery: (1) patient entering the OR, (2) first incision, (3) last suture and (4) patient leaves the OR.

Due to differences in the time of implementation of the IOR in the participating departments, data were extracted during different time periods. For the Department of Gastrointestinal Surgery, data were extracted for 6 months. For the Department of Heart, Lung and Vascular Surgery, data were extracted for 32 months. Both extraction periods were organised such that they lasted at least 4 months after installation with regard to ensuring full implementation and minimisation of start-up difficulties and learning curve effects.

The variable for surgical procedure time was analysed for (a) preparation, (b) surgery and (c) completion time, separately, as well as (d) total procedure time. These were calculated as follows: a=time between a patient entering the $\mathrm{OR}$ and first incision, $\mathrm{b}=$ time between the first incision and last suture, $\mathrm{c}=$ time between last suture and patient leaving the OR, $d=$ time between a patient entering OR and leaving OR.

In the case of missing data for all the three data time points $(\mathrm{a}, \mathrm{b}$ and $\mathrm{c})$ the surgeries were excluded. In the case of missing data for less than three data time points, we included the available registered time and the others were considered missing.

Independent t-tests were used to estimate the statistical differences in the mean times between the two OR alternatives (COR vs IOR). We tested the variance for each 


\section{Surgical procedure}

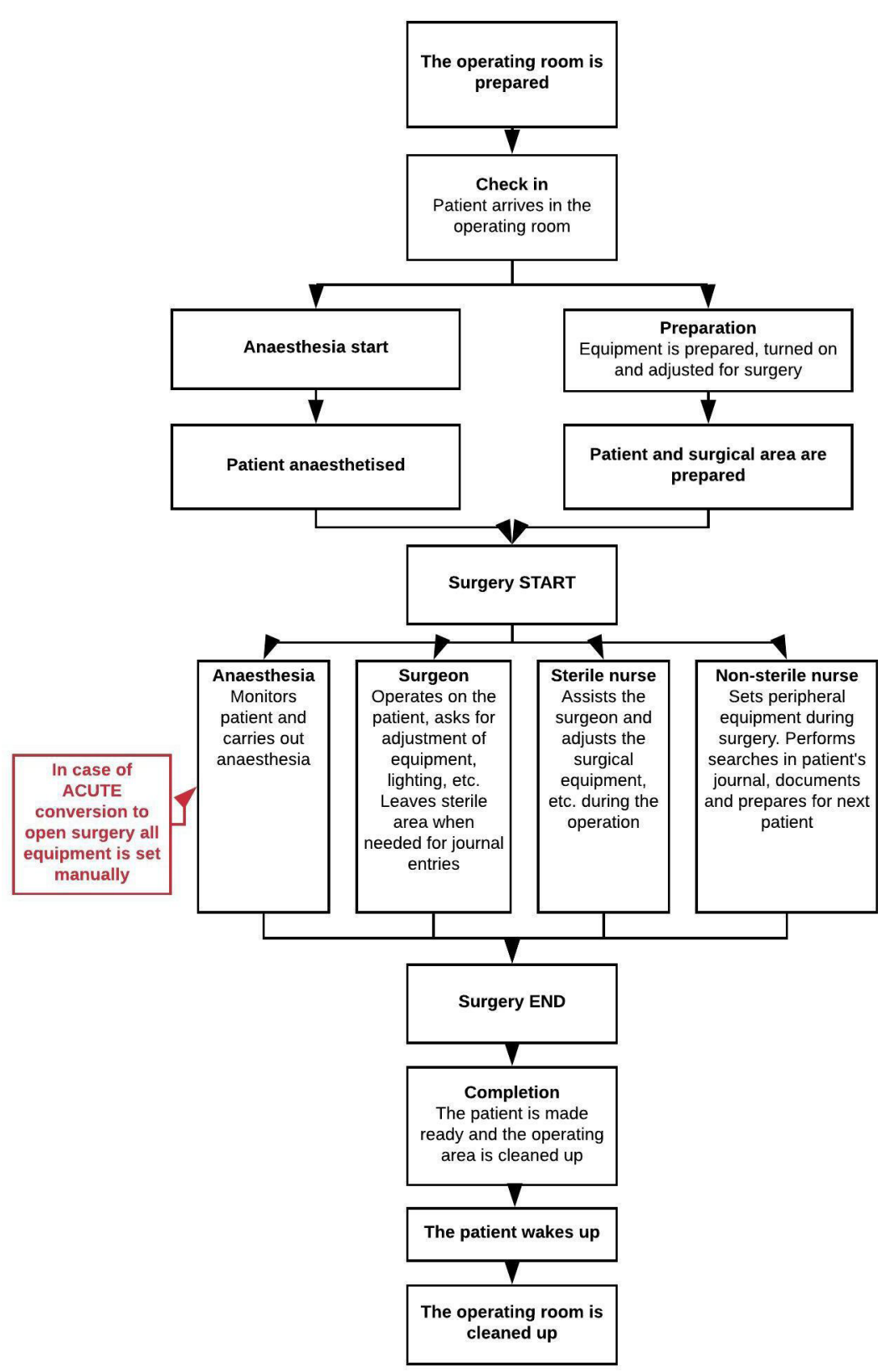

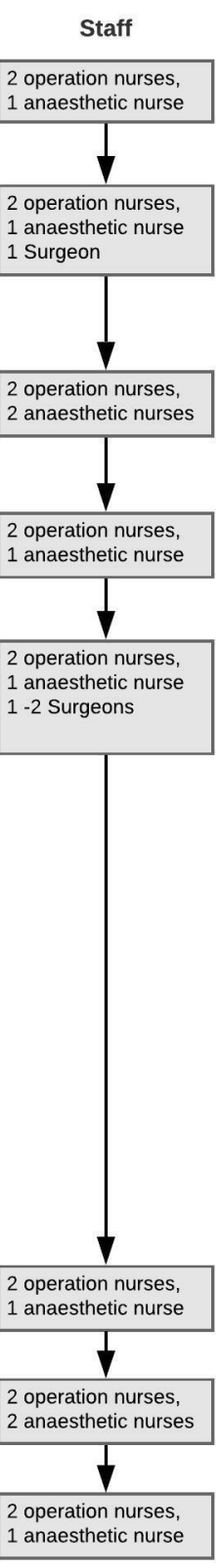

Figure 2 Surgical procedures in the conventional operating room.

surgery type and applied equal/unequal variances as appropriate.

As different surgeons presumably influence the duration of surgery, we applied a linear regression model adjusting for the influence of the surgeon to test the robustness of the results of surgery time for each surgery type. The number of different surgeons distributed by surgery type and type of OR is presented in online supplementary appendix 1 .

Sensitivity analyses were performed carrying out the analyses described above after the exclusion of outliers. Outliers were defined as data points when $<$ mean $-3 \times \mathrm{SD}$ or $>$ mean $+3 \times \mathrm{SD}$.

The analyses of time spent per patient were performed using STATA V.15. An alpha level of 0.05 was considered statistically significant.

\section{Qualitative study}

This was an explorative qualitative research study based on qualitative interviews. This design was chosen as it allows for in-depth exploration of the complexity and potential variations in the use of IORs and staff experiences of the consequences of the implementation and use of IORs. ${ }^{14-16}$

\section{Setting and recruitment}

Recruitment of interview informants was purposive $^{17}$ and based on a diverse case selection strategy in which participants were selected in order to represent variation along different relevant dimensions. ${ }^{18}$ This allowed for an exploratory investigation of different types of use and 


\section{Surgical procedure IOR}

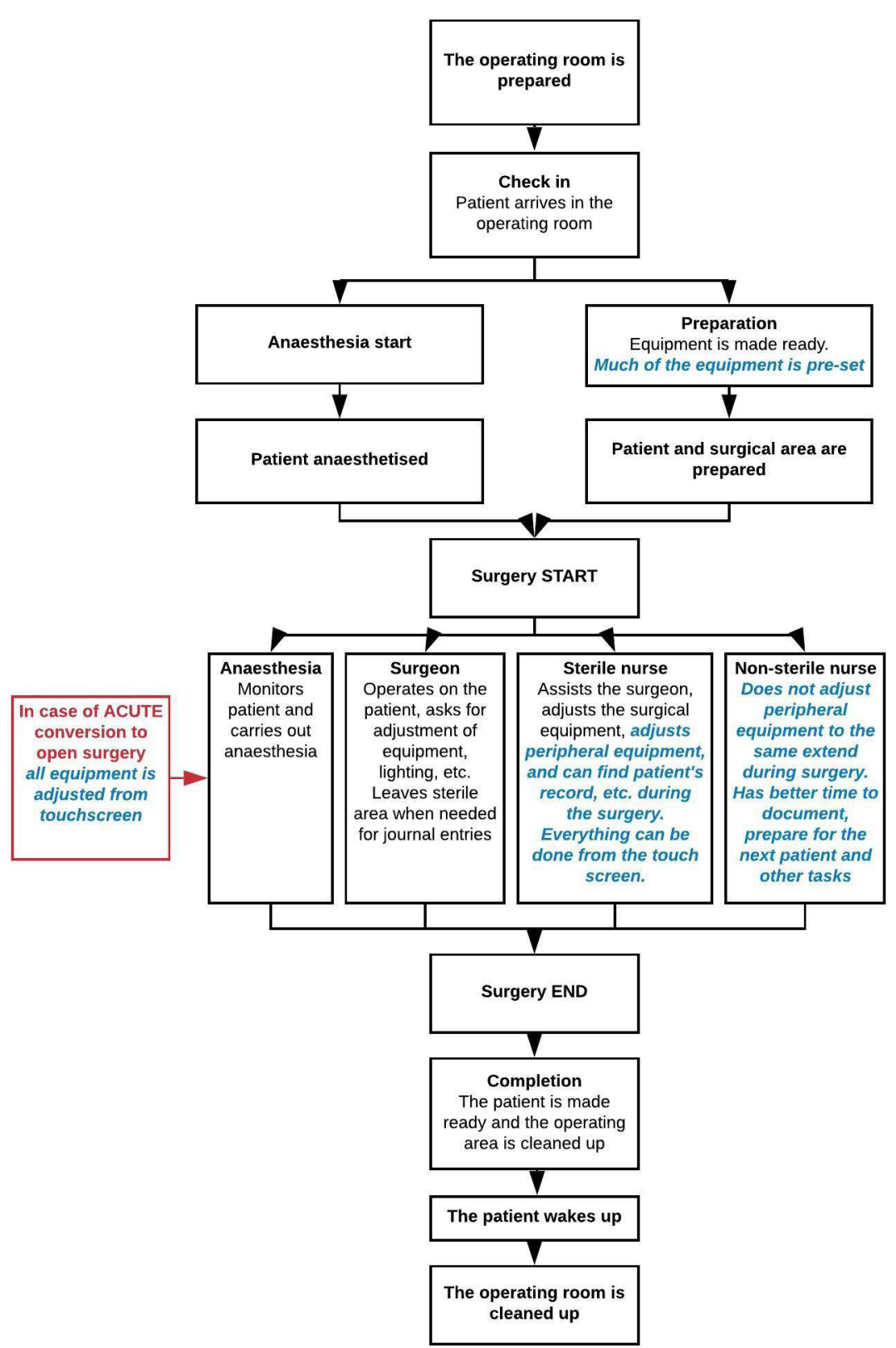

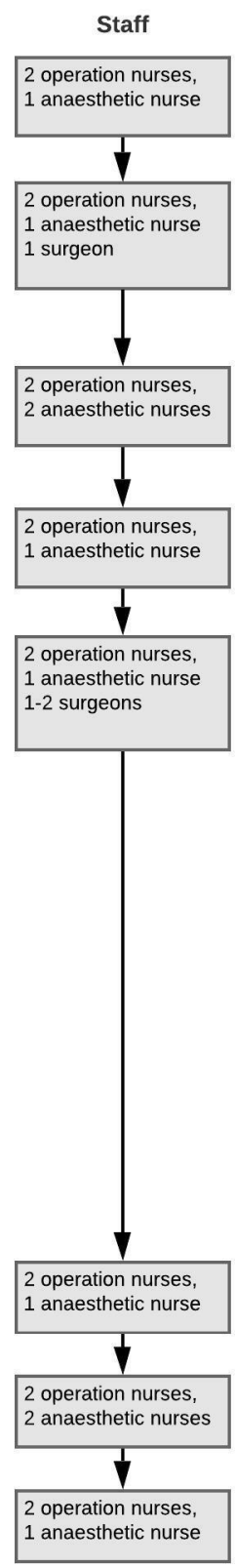

Figure 3 Surgical procedures in the integrated operating room (IOR).

experienced consequences of the implementation and use of an IOR.

We included interview informants from 10 hospital departments across seven Danish hospitals, including the two hospital departments from AUH that were included in the quantitative study. We sought to include departments from both large and smaller hospitals, and departments representing different specialities using IOR. Furthermore, we sought to include informants with different professional backgrounds (surgeons, surgical nurses and anaesthesia nurses) as they would represent different perspectives on the use of an IOR. Finally, experience with working in an IOR was a general inclusion criterion for the informants.
In the identification and recruitment of interview informants, we were assisted by medical technology advisers who manage counselling regarding medical technologies and are responsible for the purchase and implementation of medical technologies in hospitals. The advisers made the initial contact to the selected hospital departments to obtain consent for participation in the interviews and to invite the department to select and provide contact information on relevant informants for an interview. When receiving the contact information, the informants were contacted and provided with further information about the study and the interview. If the informant still wanted to participate in an interview, a time for this was scheduled. The interview informants were recruited from February to March 2019. 
Table 1 Participating departments and types of surgeries included in this study

\begin{tabular}{|c|c|c|}
\hline $\begin{array}{l}\text { Selected specifications of the } \\
\text { departments }\end{array}$ & $\begin{array}{l}\text { Department of Heart, Lung and Vascular } \\
\text { Surgery }\end{array}$ & Department of Gastrointestinal Surgery \\
\hline Full time employees & 94 & 152 \\
\hline Annual number of surgeries* & 1267 & 2627 \\
\hline Selected surgical unit & OR-East & OR-South 1 \\
\hline $\begin{array}{l}\text { Total number of ORs in the surgical } \\
\text { unit† }\end{array}$ & 5 & 3 \\
\hline $\begin{array}{l}\text { Total number of IORs in the surgical } \\
\text { unit }\end{array}$ & 1 & 1 \\
\hline $\begin{array}{l}\text { Specialty of the selected types of } \\
\text { surgery }\end{array}$ & Lung surgery & Gastrointestinal surgery \\
\hline \multirow[t]{5}{*}{ Selected types of surgery } & Lobectomy & Laparoscopic cholecystectomy \\
\hline & Oesophageal resection & Laparoscopic appendectomy \\
\hline & Pectus excavatum & \\
\hline & Lung resection & \\
\hline & Pectus carinatum & \\
\hline \multirow[t]{2}{*}{ Data extraction period } & 01.10.16-31.03.19 & 01.10.18-31.03.19 \\
\hline & 32 months $(n=1035)$ & 6 months $(n=148)$ \\
\hline
\end{tabular}

${ }^{*}$ Activity data are extracted for 2018.

†Including integrated operating rooms (IORs).

ORs, operating rooms;

In total 24 informants were recruited, and all initially agreed to participate in an interview. However, four interviews were cancelled due to unforeseen circumstances in the hospital departments. The final 20 informants included 9 surgeons, 8 surgical nurses and 3 anaesthesia nurses reflecting representation from gastrointestinal surgery (9), cardiothoracic and vascular surgery (2), gynaecological surgery (6), urological surgery (1) and paediatric surgery (2). The interview informants varied concerning their level of experience in working in IORs, ranging from a few months to several years. Interviews were concurrently transcribed, read and assessed, which allowed for the recruitment of interview informants until the authors estimated that data saturation was reached. ${ }^{19}$ Table 2 provides an overview of the conducted interviews.

\section{Data collection and analysis}

The interviews were conducted as telephone interviews $(n=9)$ or performed face-to-face $(n=11)$ depending on the preferences of the interview informants between March and April 2019. The interviews were conducted by two of the authors responsible for the qualitative study, who are both experienced social researchers and not affiliated with the hospitals. The two authors conducted the first two interviews together to ensure consistency across the subsequent interviews that were then conducted individually.

The interviews were guided by semistructured interview guides with open-ended questions. ${ }^{15}$ The interview guides covered the following themes:
- Differences and changes in the working procedures and inter-professional cooperation between the CORs and IORs that were identified in phase I (see figure 3). Example questions included 'Can you explain for me how the work processes in relation to the surgery of patients are different in the IOR compared with the COR?' and 'Which changes have you experienced from the use of the IOR that have impacted on your inter-professional cooperation and communication in the OR?'.

- Staff and education requirements and training processes for using the IOR. Example questions included 'What requirements in relation to staff and education are required for using the IOR?' and 'How is the training of staff (surgeons/nurses) in using the IOR organised?'.

- Experienced consequences of using the IOR compared with COR. Example questions included 'What challenges do you experience when using the IOR compared with the COR?' and 'What possibilities arise when using the IOR compared with COR?'.

The questions asked were modified when needed in relation to the informants' professional background (ie, surgeon or nurse). During the interviews, staff were encouraged to speak freely and the interviewer asked follow-up questions to remove any potential ambiguity and to ensure the correct understanding of the answers given. The interviews lasted approximately $30 \mathrm{~min}$, and all were digitally recorded and transcribed verbatim. 
Table 2 Overview of interview informants

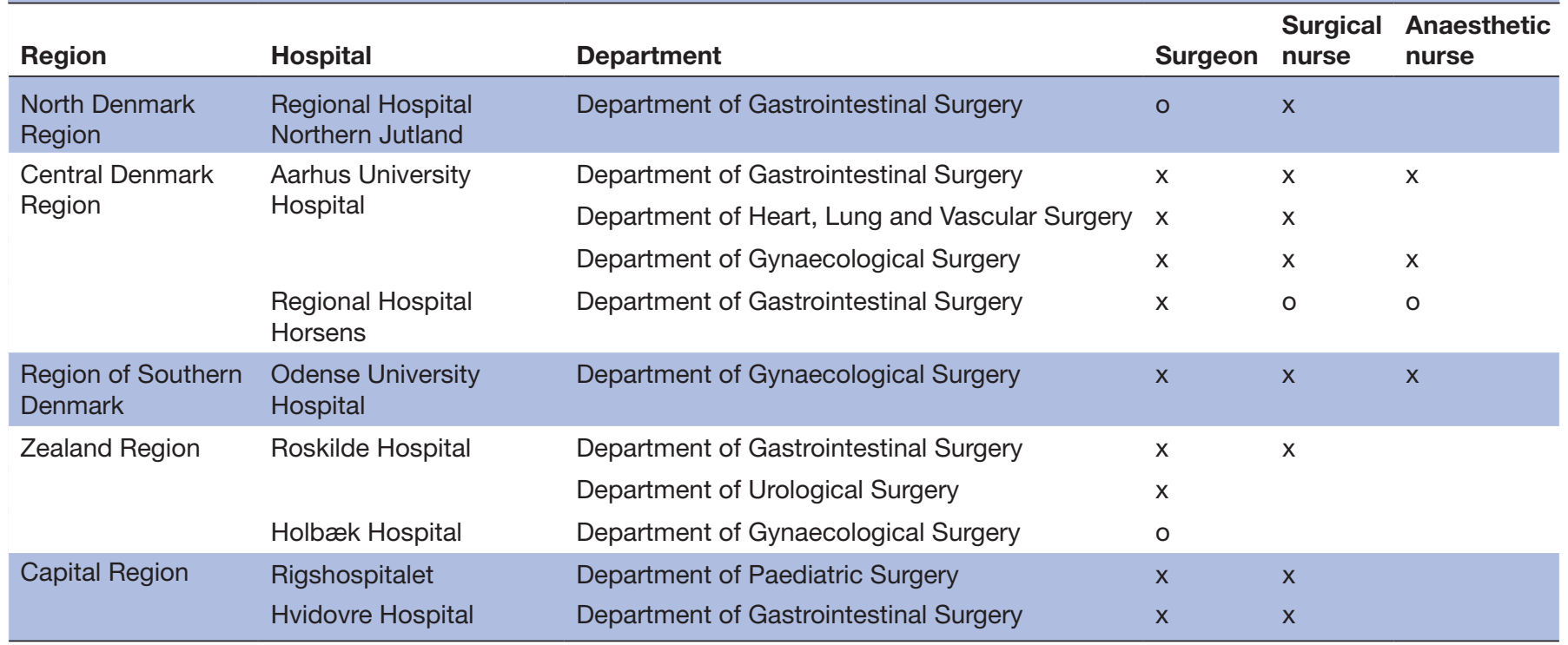

$x$, participated in an interview.

o, invited for participation but an interview did not take place.

Empty space indicates not invited for interview.

The transcribed data from the interviews were analysed using Thematic Analysis ${ }^{20}$ and comprised the following steps. First, all interviews were thoroughly read and re-read by the two authors conducting the interviews to generate the initial codes. Three interviews were test-coded independently by the two authors and any discrepancies in coding were discussed and resolved by consensus. Subsequently, all interviews were coded by one of the two authors using NVIVO V.11.0 (QSR International, Melbourne, Australia). The codes were read and sorted into main themes and subthemes which were discussed by the authors and subsequently refined. The themes were then investigated in the full dataset, looking for inconsistent evidence. ${ }^{14} 17$ Following this process, the final themes were defined (see the Results section).

\section{Patient and public involvement}

This research was conducted without patient involvement as it was not appropriate or possible to involve patients or the public in the design, conduct, reporting or dissemination of our research.

\section{RESULTS}

\section{Quantitative study}

During the quantitative study, a total of 1192 surgeries of the selected types were performed in the ORs. Nine surgeries were excluded before analysis due to having missing data at all three time points.

The results of the statistical analyses are shown in table 3. The preparation time for lobectomy was found to be significantly lower and completion time for cholecystectomy was significantly higher in the IOR compared with COR. We found no other statistically significant differences in preparation time, surgical time, completion time or procedure time between the IOR and COR. However, we saw a slight tendency towards preparation time being lower in the IOR compared with COR. Regression analyses adjusting for the potential influence of the surgeon on surgery time did not change these results. Due to the very low sample size, the pectus carinatum surgery type did not meet the conditions for the regression and was therefore excluded from these analyses.

To test the robustness of the results, we performed sensitivity analyses excluding outliers. An overview of the included data in the different time calculations, missing data and excluded outliers is available in online supplementary appendix 2. Overall, the sensitivity analyses did not change the results. Only the surgery time for lung resection appeared to be significantly higher in the IOR compared with the COR when performing the regression analyses adjusting for the influence of a specific surgeon. The results of the sensitivity analyses are presented in online supplementary appendix 3 .

\section{Qualitative study}

The thematic analysis resulted in the identification of four themes related to the differences in procedures between the IOR and COR, and the consequences of implementing and using the IOR as experienced by staff. These included: (1) consequences of the IOR for current work processes and work tasks, (2) impact of the IOR on cooperation and communication, (3) impact on the experienced workflow during surgery and (4) impact on ergonomics and working conditions. While we understand 


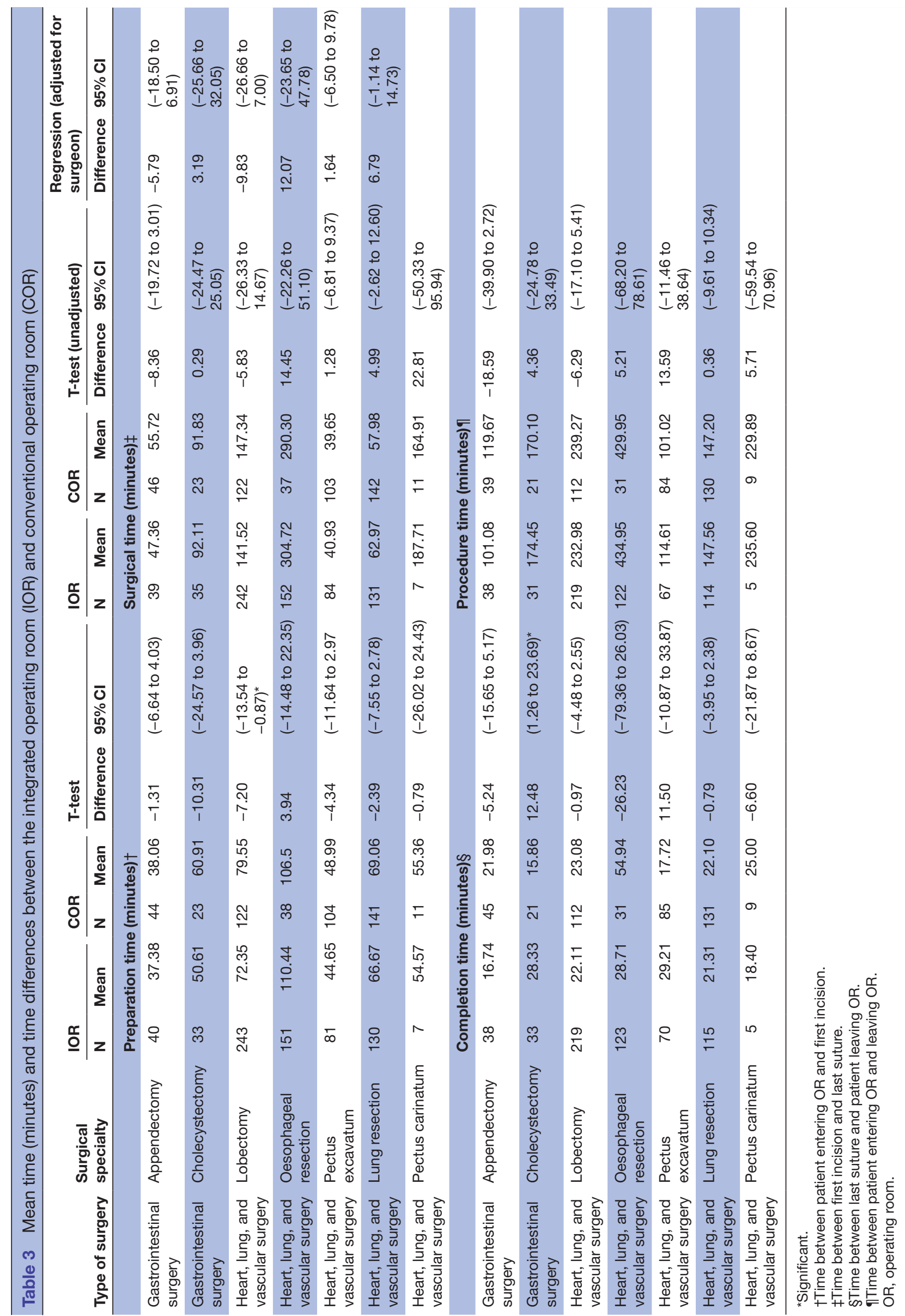


work processes as relating to the actual differences and changes in how things are done between the IOR and COR, for example, in terms of procedures, tasks among the personnel and division of labour, we understand work flow as the experienced dimension of the differences and changes in the work processes.

The qualitative interviews showed that, in most surgical departments, the surgical nurses were responsible for administering the touchpad and thus the integrated equipment and functionalities. Most IORs had a touchpad placed in both the sterile and non-sterile field, which allowed both the sterile and non-sterile nurses to administer the technology. As a consequence, variation was found concerning which of the nurses administered the touchpad. This was highly dependent on the habits and preferences among nurses, although the touchpad was most commonly administered by the sterile nurses.

The interviews showed that when the touchpad was administered by the sterile nurse, use of the IORs led to a change in the division of labour between the sterile and non-sterile nurse, compared with the CORs. Administration of the touchpad allowed the sterile nurse to control all the IOR equipment and functionalities, which in the CORs were controlled by the non-sterile nurse. This change provided the sterile nurse with greater autonomy and made the surgical team less dependent on the nonsterile nurse in the administration of the equipment and functionalities during surgery. Simultaneously, the nonsterile nurse experienced a release of time resources and fewer disruptions while handling other tasks during surgery (eg, documentation, preparation of surgical equipment and preparation of material for pathologists). No non-sterile nurses reported that they handled any new tasks in the IOR compared with the COR. Similarly, the sterile nurses did not report experiencing an increased number of disruptions or lack of time in handling their tasks during surgery as a result of the change in the division of labour. In situations where the touchpad was administered by the non-sterile nurse, the surgical staff experienced no changes in the work processes and division of labour during surgery compared with the COR.

Across hospitals, departments and staff, the main experienced advantage of the IORs was improvements to the workflow during surgery. Generally, both surgeons and surgical nurses experienced that the IORs facilitated more smooth and efficient working procedures in terms of faster response on requests for adjustments of surgical equipment and functionalities and fewer disruptions during surgery. However, the interviews showed that realisation of the workflow improvements presupposed either administration of the touchpad in the sterile field or flexible administration of the touchpad. Flexible administration meant that the sterile personnel administered the touchpad, at least when the non-sterile nurse was occupied or not present in the OR.

Furthermore, the interviews indicated that improvements to workflow were particularly apparent during surgical procedures of a longer length and greater complexity, as the need for adjustments in surgical equipment and functionalities were greater in such procedures compared with shorter and less complex procedures. This resulted in some surgeons and nurses stressing that IORs have the greatest potential in relation to complex and lengthy surgical procedures. Only a small number of the interviewed surgeons and nurses reported that they did not experience any improvements in the workflow in IORs compared with CORs.

Generally, the use of IORs was not experienced to significantly affect cooperation among staff in the OR. Thus, all professional groups experienced interdisciplinary cooperation in the IOR to be very similar to that in the COR. A small number of sterile nurses reported that they became more involved in the surgical procedure in the IOR compared with in the COR due to closer communication with the surgeon when they were administering the touchpad.

A final consequence of using IORs compared with COR was related to working conditions and ergonomics. Several non-sterile nurses mentioned that the use of IORs led to a better working environment and better ergonomics compared with CORs. These nurses emphasised that this improvement was due to better working postures, reduction of risk of falling over cables and less walking about during surgery. The improvement was thus a consequence of administering the IOR equipment and functionalities from the touchpad, and the changed division of labour between the sterile and non-sterile nurse. Neither the sterile nurses nor the surgeons generally experienced any improvements in working conditions or ergonomics as a result of the IOR, and they did not mention worsened working conditions or ergonomics.

\section{DISCUSSION}

In phase I, we identified no measurable clinically important changes in the safety, quality or patient outcomes resulting from the implementation and use of IORs. Therefore, these kinds of effectiveness outcomes were not included in the next phase of the study. Phase II consisted of a quantitative study addressing the outcomes relating to time spent on each patient and a qualitative study investigating the staff experiences of the identified differences in procedures between the CORs and IORs.

In the quantitative study, preparation time for lobectomy was found to be significantly lower and completion time for cholecystectomy was significantly higher in the IOR compared with COR. The interviews revealed that staff experienced the IOR as leading to improved workflow and that although the IOR was considered a practical technological solution it did not have a major impact on daily work.

According to the qualitative study, staff experienced that the IOR led to improved workflow during surgery resulting from faster responses to requests for adjustments to the surgical equipment and functionalities, presupposing that the touchpad was administered either 
entirely in the sterile field or flexibly between the sterile and non-sterile field. This positive impact of IORs on workflow is supported by several studies emphasising how IORs contribute to improvements to work processes during surgical procedures. ${ }^{21-24}$ Moreover, some sterile nurses mentioned better cooperation with the surgeon as a result of administrating the touchpad. This outcome is supported by findings from Stavroulis $e t a l^{23}$ who identified better teamwork between surgeons and sterile nurses as an experienced advantage of IORs. It is expected that improved workflow and teamwork translate into positive effect on performance outcomes such as effectiveness, patient safety and efficiency within different healthcare settings relating to ORs, ${ }^{25}$ however a review by Tan $e t$ al found no studies that objectively demonstrated that team skills resulted in improved patient outcomes. ${ }^{26}$ Previous studies by Blikkendal $e t$ al and Strauss $e t$ al ${ }^{12}{ }^{27}$ suggested that an improvement of workflow would result in reduced procedure times in ORs. However, we did not observe such an effect and suggest that the positive effects of the experienced improvements may occur over time and on other parameters.

Improved ergonomics and working conditions for surgical personnel attributable to IORs have been only sparsely addressed in the literature. In a few studies, the IOR was stated to contribute to a reduction of the stress levels among surgical personnel during surgery due to the ease in control and adjustment of surgical equipment provided by the touchpad. ${ }^{21}{ }^{23}$ Perceived stress has recently been found to be associated with risk of absence due to sickness, suggesting that reduced stress among surgical personnel might have a positive effect on absence over time. ${ }^{28}$ In our study, the non-sterile nurses experienced an improvement to their working environments following the implementation and use of the IOR.

In summary, clinical personnel generally perceived the IOR as a technology that did not require major changes to the organisation or workflow in the OR. The IOR was considered to be a practical technological solution but one without having a major impact on daily work.

Beyond the findings of this study, other consequences or value of the IOR may be found in future research. Positive effects may be identified to both time and safety when switching from laparoscopic to open surgery in acute situations (not measured here due to the low frequency of occurrence) as well as during complex and lengthy procedures. The extended use of teaching and collaborating possibilities due to video streaming may also ensure the efficient and continuous education of medical students and surgeons, thereby improving the quality of treatment.

As such, there is a need for future research to systematically integrate the analysis of organisational aspects with more objective quality and performance indicators, such as patient safety and quality in treatment, preferably using an experimental design linking the qualitative and quantitative data to substantiate conclusions on consequences of IORs. Additionally, future research could usefully address the costs or cost-effectiveness of IORs compared with
CORs in order to inform decisions regarding whether to fund IORs within the healthcare system.

\section{Strengths and limitations of the study}

The relatively large dataset was a strength of the quantitative study. However, there were very few observations in some types of surgeries which resulted in larger CIs and larger statistical uncertainty.

Due to the different times of implementation of the IORs, periods of data extraction differed by departments studied. Data extraction was performed 4 months after installation with regard to ensuring full implementation and minimising start-up challenges. However, as the IOR was relatively recently implemented in the Department of Gastrointestinal Surgery, less data were available for these surgery types, as well as the potential influence of learning curve effects being greater than for the Department of Heart, Lung and Vascular surgery, where the IOR had been implemented for a longer period of time. These time data were extracted from only one hospital, which further limits the generalisability of the results. Possible time savings are closely related to operating procedures that may vary among settings.

The quality of the data is closely related to the accuracy of the information provided, and this information may be subject to bias, given that the data were recorded manually by the non-sterile nurse in the OR. Another bias may be introduced by the lateness of physicians, although this was only related to 'preparation time'. However, these potential biases are expected to be randomly distributed among the CORs and IORs, and thus, of limited importance for our results.

If, as our interviews suggested, that IORs are experienced as better or safer among staff, systematic differences between the ORs might occur if complicated procedures are systematically allocated to IORs. This would result in selection bias and could lead to an underestimation of time differences between the ORs, as complicated procedures are expected to be more time consuming than simple procedures.

We adjusted the analyses of surgery time to account for the influence of the surgeon but this did not change our results. Other factors, such as patient age, sex and comorbidities could potentially have influenced the results but due to lack of data for these factors, it was not possible to include these variables in the regression analyses.

A key strength of the qualitative interview study was the relatively large and varied sample with representatives from different regions, hospitals, specialities and professional backgrounds. This, together, with high-quality data collection (due to the researchers being experienced social scientists and not affiliated with the hospitals) and the researchers engaging in regular discussions about data saturation during data collection, all contributed to this research having high informational power and internal validity. ${ }^{15} 18$

The substantial qualitative data collection including additional settings than the operating departments from 
which the quantitative data were extracted might be a limitation of the study as it introduces variability. Linking the qualitative and quantitative data to the same location, time, setting and surgeries would reduce such variability and enhance the validity of results.

According to Danish guidelines, two nurses (one sterile and one non-sterile) have to be present in the OR throughout each surgery. Such guidelines may differ among countries and influence the organisation and realisation of potential advantages resulting from a change in the division of labour. Consideration of the external validity of the results thus needs to take into account that their applicability may be limited to countries with similar organisation and processes within hospitals. However, by making the organisation and processes within the IORs and CORs explicit and transparent, readers can hopefully assess the relevance and applicability of these results to their own context.

\section{CONCLUSIONS}

According to the qualitative study, staff experienced that the IOR led to improved workflow during surgery resulting from faster responses to requests for adjustments to surgical equipment and functionalities. However, this improved workflow did not translate into reduced procedure time compared with the COR, as only one time point for one surgery type was found to be statistically significant reduced. Some sterile nurses indicated they had experienced better cooperation with surgeons as a result of administrating the touchpad in the sterile field and several of the non-sterile nurses expressed that the IOR improved their working environment and ergonomics. No negative consequences were identified by the interviewed staff as a result of using IORs compared with CORs. Overall, the IOR was considered a practical technological solution although it did not have a major impact on daily work. Thus, in this study, the IORs were generally not experienced to lead to major improvements or associated with important clinical advantages compared with the CORs.

Acknowledgements The authors thank the clinical staff of all participating hospital departments for their assistance and time in relation to the observational visits and interviews, and their guidance on comparable types of surgery. The authors also thank the Data Unit at Aarhus University Hospital for their help in accessing the relevant quantitative data.This study was performed as a part of a full Danish health technology assessment: 'Integrated Operating Rooms-A Health Technology Assessment', which was conducted on behalf of the Danish Health Authorities. Therefore, the authors also want to thank the project group and stakeholders for their valuable contributions and supply of essential information.

Contributors $\mathrm{KC}$ developed the study, designed the qualitative data collection and planned and conducted interviews with clinical staff. She performed the qualitative analysis and contributed to drafting the manuscript. EKJ made substantial contributions to both the qualitative and quantitative data collection. She conducted interviews with clinical staff, and she performed the analyses and contributed to drafting the manuscript. MLM, AMLT, CL and NTD contributed to the conception and study design. They critically commented on the analyses and the manuscript. BWR developed the study, designed the quantitative data collection and planned the quantitative data collection. She performed the quantitative analysis and contributed to drafting the manuscript. All authors approved the final manuscript.
Funding The research was supported by Aarhus University Hospital and the five Danish regions.

Competing interests None declared.

Patient consent for publication Not required.

Ethics approval The data collection for this study was registered and approved by the Danish Data Protection Agency (Journal no. 1-16-02-322-19). According to Danish law, this study did not require ethics approval from the National Committee on Health Research Ethics as it was an interview study and because the study did not involve personally sensitive information or human material. All informants/ participants gave informed consent before taking part in the interviews.

Provenance and peer review Not commissioned; externally peer reviewed.

Data availability statement No data are available. The raw data represent confidential information and cannot be made public available according to the specific data license agreement (granted solely for the purpose of the project evaluation). The Stata software code is available upon request.

Open access This is an open access article distributed in accordance with the Creative Commons Attribution Non Commercial (CC BY-NC 4.0) license, which permits others to distribute, remix, adapt, build upon this work non-commercially, and license their derivative works on different terms, provided the original work is properly cited, appropriate credit is given, any changes made indicated, and the use is non-commercial. See: http://creativecommons.org/licenses/by-nc/4.0/.

ORCID iD

Bettina Wulff Risør http://orcid.org/0000-0003-3701-074X

\section{REFERENCES}

1 Haynes AB, Weiser TG, Berry WR, et al. A surgical safety checklist to reduce morbidity and mortality in a global population. $N$ Engl J Med 2009;360:491-9.

2 Gallagher AG, Smith CD. From the operating room of the present to the operating room of the future. Human-factors lessons learned from the minimally invasive surgery revolution. Semin Laparosc Surg 2003;10:127-39.

3 Catchpole K, Mishra A, Handa A, et al. Teamwork and error in the operating room: analysis of skills and roles. Ann Surg 2008;247:699-706.

4 Vincent C, Moorthy K, Sarker SK, et al. Systems approaches to surgical quality and safety: from concept to measurement. Ann Surg 2004;239:475-82.

5 Sundhedsplanlægning, Koncern HR Fysisk Arbejdsmiljø. Designguide for hospitalsbyggeri I region Midtjylland. Version II. Region Midtjylland, 2018.

6 Golatowski F, Janss A, Leucker M, et al. OR.NET - secure dynamic networks in the operating room and clinic. Biomed Tech 2018;63:1-3.

7 Buzink SN, van Lier L, de Hingh IHJT, et al. Risk-sensitive events during laparoscopic cholecystectomy: the influence of the integrated operating room and a preoperative checklist tool. Surg Endosc 2010;24:1990-5.

8 Perrakis A, Hohenberger W, Horbach T. Integrated operation systems and voice recognition in minimally invasive surgery: comparison of two systems. Surg Endosc 2013;27:575-9.

9 Salama IA, Schwaitzberg SD. Utility of a voice-activated system in minimally invasive surgery. $J$ Laparoendosc Adv Surg Tech A 2005;15:443-6.

10 Luketich JD, Fernando HC, Buenaventura PO, et al. Results of a randomized trial of HERMES-assisted versus non-HERMES-assisted laparoscopic antireflux surgery. Surg Endosc 2002;16:1264-6.

11 Hüfner T, Citak M, Imrecke J, et al. [Handling modern imaging procedures in a high-tech operating room]. Unfallchirurg 2012;115:220-5.

12 Blikkendaal MD, Driessen SRC, Rodrigues SP, et al. Surgical flow disturbances in dedicated minimally invasive surgery suites: an observational study to assess its supposed superiority over conventional suites. Surg Endosc 2017;31:288-98.

13 Wisdom J, Creswell JW. Mixed methods: integrating quantitative and qualitative data collection and analysis while studying patientcentered medical home models. Rockville, MD: Agency for Healthcare Research and Quality, 2013.

14 Maxwell JA. Qualitative research design: an interactive approach. Thousand oaks,CA: SAGE Publications, 2012.

15 Kvale S, Brinkmann S. Interviews: learning the craft of qualitative research interviewing. Los Angeles.CA: SAGE Publications, 2009. 
16 Spradley JP. The ethnographic interview. Australia: Wadsworth Group/Thomson Learning, 1979.

17 Patton MQ. Qualitative research \& evaluation methods. Thousand Oaks,CA: SAGE Publications, 2002.

18 Seawright J, Gerring J. Case selection tecniques in case study research: a menu of qualitative and quantitative options. Political Research Quarterly 2008;61:294-308.

19 Malterud K, Siersma VD, Guassora AD. Sample size in qualitative interview studies: guided by information power. Qual Health Res 2015;26:1753-60.

20 Braun V, Clarke V. Using thematic analysis in psychology. Qual Res Psychol 2006;3:77-101.

21 Nocco U, del Torchio S. The integrated or efficiency and effectiveness evaluation after two years use, a pilot study. Int $J$ Comput Assist Radiol Surg 2011;6:175-86.

22 Rockstroh M, Franke S, Hofer M, et al. OR.NET: multi-perspective qualitative evaluation of an integrated operating room based on IEEE 11073 SDC. Int J Comput Assist Radiol Surg 2017;12:1461-9.
23 Stavroulis A, Cutner A, Liao L-M. Staff perceptions of the effects of an integrated laparoscopic theatre environment on teamwork. Gynecol Surg 2013;10:177-80.

24 Koninckx PR, Stepanian A, Adamyan L, et al. The digital operating room and the surgeon. Gynecol Surg 2013;10:57-62.

25 Buljac-Samardzic M, Doekhie KD, van Wijngaarden JDH. Interventions to improve team effectiveness within health care: a systematic review of the past decade. Hum Resour Health 2020;18:2.

26 Tan SB, Pena G, Altree M, et al. Multidisciplinary team simulation for the operating theatre: a review of the literature. ANZ J Surg 2014;84:515-22.

27 Strauss G, Aries F, Abri O, et al. [Conception, realization and analysis of a modern operating theatre workplace for ENT surgery]. HNO 2010;58:1074-84.

28 Thorsen SV, Pedersen J, Flyvholm M-A, et al. Perceived stress and sickness absence: a prospective study of 17,795 employees in Denmark. Int Arch Occup Environ Health 2019;92:821-8. 